\title{
Axillary mass in a woman with progressive exertional limbs numbness and fatigue: a case report
}

\author{
Xiaoliang Sun, Jun Liu, Meng Yang, Linping Huang \\ Department of General Surgery, China-Japan Friendship Hospital, Beijing, China \\ Correspondence to: Linping Huang, MD. Department of General Surgery, China-Japan Friendship Hospital, Rd. Yinghuayuandongjie, Chaoyang \\ District, Beijing, China. Email: huanglinping2020@163.com.
}

\begin{abstract}
Occult breast cancer (OBC) is a very rare type of breast cancer which accounts for only $0.2 \%$ to $0.9 \%$ of all breast cancer defined as histologically proven breast cancer found from outside the breast especially axillary with the absence of a primary tumor in the breast. With the development of highresolution ultrasound, mammography, especially breast-enhanced MRI, the incidence of OBC has decreased in recent years. A standard approach for OBC is to perform a modified radical mastectomy (MRM) at the time of axillary lymph node dissection (ALND). The five-year survival rates after treatment of an occult primary breast cancer with axillary metastases range from $59 \%$ to $93 \%$, averaging about $75 \%$. Paraneoplastic neurological syndromes (PNS) are a very rare heterogeneous group of disease caused by mechanisms of the remote effects of malignant tumors other than tumor metastases, infections or side effects of tumor treatment. Malignant tumors with PNS account for only less than $0.01 \%$ of all tumors. The treatment of PNS include removal of the tumors and suppression of the immune response. The prognosis of PNS depends on the degree of neuronal damage during the treatment of the disease. PNS is a rare neurological complication in patients with breast cancer and particularly rare in the patients with OBC. Here, we report a rare case of a 62-year-old woman diagnosed as OBC who presented with PNS as the first symptom.
\end{abstract}

Keywords! Occult breast cancer (OBC); paraneoplastic neurological syndromes (PNS); axillary mass

Submitted Oct 25, 2020. Accepted for publication Jan 29, 2021.

doi: $10.21037 /$ gs-20-780

View this article at: http://dx.doi.org/10.21037/gs-20-780

\section{Introduction}

Paraneoplastic neurological syndromes (PNS) are an aberrant immune-mediated neurological disorders caused by mechanisms of the remote effects of malignant tumors other than metastases, metabolic or infections. The whole body nervous system will be affected by these disorders (1). The malignant tumor may be small and difficult to detect (2).

Occult breast cancer (OBC) is a very rare type of breast cancer defined as the presence of histologically proven breast cancer from axillary metastasis with the absence of a primary tumor in the breast. In modern series, OBC accounts for $0.1 \%$ to $0.8 \%$ of all newly diagnosed breast cancers (3), and the incidence has not decreased with improvements in breast imaging (4).

Both PNS and OBC are very rare in clinical practice.
To our knowledge, this is the first case reporting an OBC patient with PNS as the first symptom. Our case report should promote notification and vigilance by physicians of other cases who presents PNS as the first symptom of breast cancer. We present the following case in accordance with the CARE reporting checklist (available at http://dx.doi. org/10.21037/gs-20-780).

\section{Case presentation}

A 62-year-old patient presented with progressive exertional limbs numbness and fatigue for 9 months without other symptoms. She was diagnosed as inflammatory demyelinating polyradiculoneuropathy in the local hospital as her electromyography demonstrated neurogenic injury (EMG examination suggested peripheral nerve injury, 
mainly involving sensory nerves, axonal myelin injury, mainly axonal injury, and the sural nerve biopsy considered the pathological changes of sensory neuron neuropathy) and treated with gamma globulin, methylprednisolone and prednisone. However, the patient was sent to our hospital 1 month later for worsened symptoms and onset of new symptoms of headache, arthralgia and hypoesthesia. The myodynamia of proximal-end of upper limbs were normal, but the distal-end was grade I-II; while the proximal-end of lower extremities dropped to grade IV, but the distalend was normal. Skin hypoalgesia was also found in the extremities. The pathologic signs were negative. The patient denied any past medical history and family history.

Laboratory investigations of blood samples, fecal occult blood tests, hepatic and renal function tests, and tests for tumor markers such as alpha fetoprotein (AFP), cancer antigen 125 (CA125), carbohydrate antigen 19-9 (CA199) and carcinoembryonic antigen (CEA) were normal. The autoantibodies in the blood such as antinuclear-nuclear antibody, anti-Sm antibody were negative. The onconeural antibodies such as anti-Hu, PNMA2, CV2, Ri, amphiphysin and Yo were all negative too.

A round mass was found in the left axilla with a diameter of $3 \mathrm{~cm}$ and hard texture which is difficult to move during the physical examination. The mammography found no masses and signs of malignant calcification in the breasts. Ultrasound found an irregular shape mass in the left axilla but no abnormalities in the breasts. The mass was located at the top of the axilla which was not scanned by the mammography. The PET-CT scan demonstrated high perfusion and hypermetabolism of glucose in the mass (Figure 1).

Pathological findings of the needle biopsy of the left axillary mass indicated metastatic lymph nodes from breast cancer. The lymph node was resected and pathological immunohistochemistry (IMH) findings showed: E-cadherin (+), ER (strongly positive, 95\%+), HER-2(4B5) (-), Ki-67 (+), Mammaglobin (-), P120 (membrane+), PR (strongly positive, $90 \%+$ ).

Modified radical mastectomy (MRM) for breast cancer was performed under general anesthesia. Post-operative pathological findings showed in situ micro-focal ductal carcinoma (two foci, with diameter $\leq 1 \mathrm{~mm}$ ), with no other axillary lymph node metastasis (0/23). Adjuvant hormone therapy was started after surgery and continued for five years without chemotherapy and radiotherapy. Patient's neurological symptoms were significantly alleviated after surgery and no recurrence or metastasis was found after
5 years' follow-up.

All procedures performed in studies involving human participants were in accordance with the ethical standards of China-Japan Friendship Hospital and with the Helsinki Declaration (as revised in 2013). Written informed consent was obtained from the patient.

\section{Discussion}

PNS are a heterogeneous disorders caused by mechanisms of the remote effects of malignant tumors other than tumor metastases, infections or tumor treatments. Malignant tumors with PNS account for only less than $0.01 \%$ of all tumors (5). PNS diagnosis may precede a cancer diagnosis. Paresthesia and distal weakness caused by nerve damage are the most common clinical manifestations in patients with sensorimotor neuropathy (6). The etiology of sensorimotor neuropathy is various and complicated, and it is often difficult to diagnose. PNS should be considered when other possible causes are ruled out (7). The treatment of PNS include removal of the antigen source by treatment of the underlying tumors and suppression of the immune response. The oncologic treatment and immunotherapy can be beneficial. If the neurologic syndrome isn't relieved after treatment, it may be due to irreversible neuronal damage that occurred before the diagnosis was made and treatment begun. OBC was first recognized by William Halsted in 1907, who described three patients presenting with axillary masses that were eventually found to represent breast cancer (8). $\mathrm{OBC}$ is a type of cancer which accounts for only $0.2 \%$ to $0.9 \%$ of all breast cancer (9) defined as histologically proven breast cancer found from outside the breast especially axillary with the absence of a primary tumor in the breast. The five-year survival rates after treatment of OBC with axillary metastases range from $59 \%$ to $93 \%$, averaging about $75 \%(4,10,11)$. PNS is a rare neurological complication in patients with breast cancer and particularly rare in the current case with $\mathrm{OBC}$.

This case has illustrated the possibilities of OBC presenting with extremities numbness and fatigue. The diagnosis of PNS was achieved by differential diagnosis. And the OBC was further found with physical examination of axillary mass while no tumor was found in breast by imaging modalities. Awareness of this kind of disease can assist physicians in raising clinical suspicion of underlying causative malignancy, thus performing a proper and thorough examination, arriving at a correct diagnosis, and 

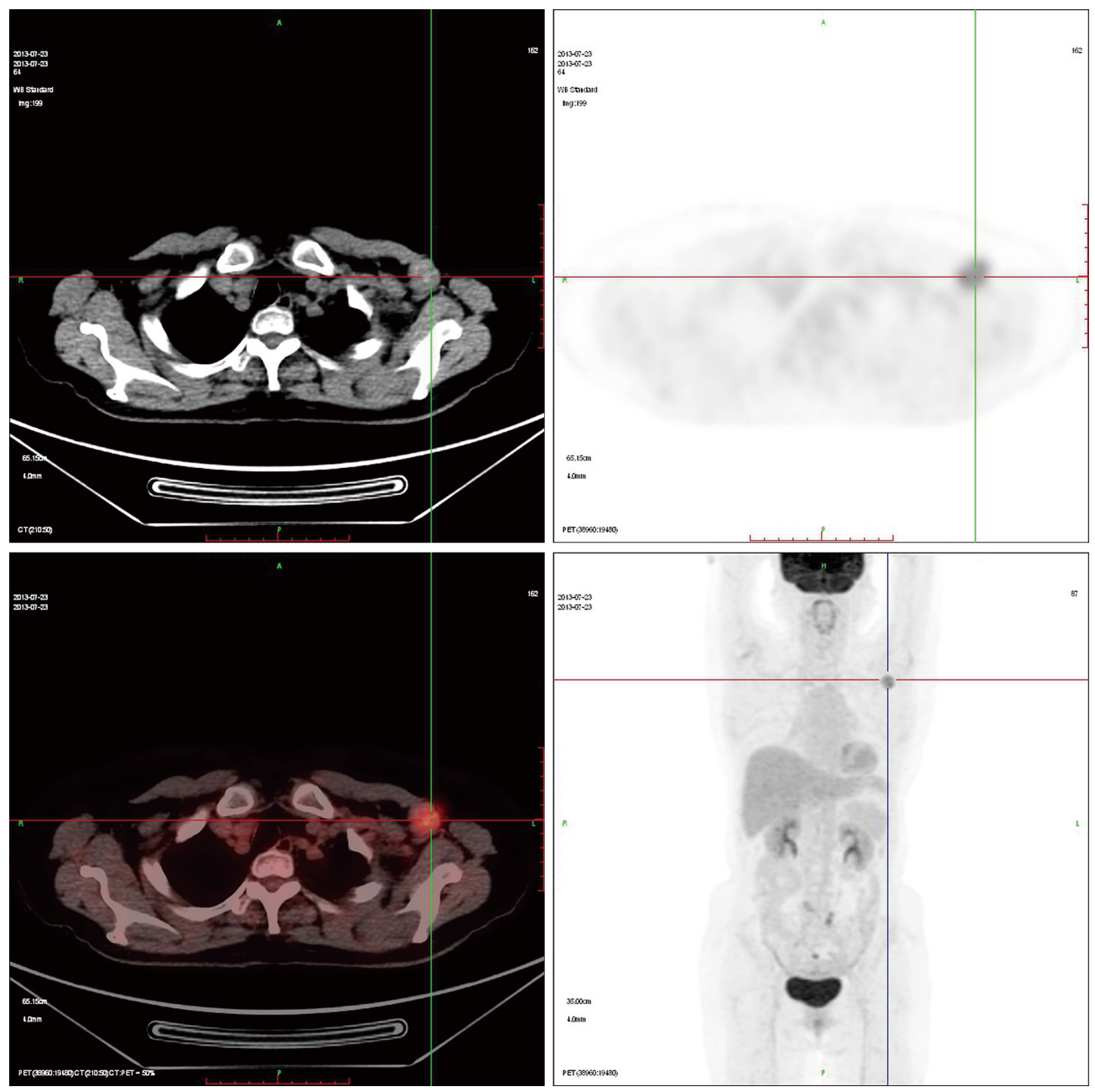

Figure 1 Positron emission tomography-computed tomography (PET-CT) shows an enlarged lymph node $($ size, $2.4 \mathrm{~cm} \times 2.4 \mathrm{~cm} \times 2.2 \mathrm{~cm})$ with highly radioactive concentration in the left axilla with irregular shape and uneven density, and the standardized uptake value (SUV $\left.\mathrm{V}_{\text {max }}\right)$ is 6.3. No abnormalities are found in the breast.

determining appropriate treatment.

\section{Acknowledgments}

Funding: None.

\section{Footnote}

Reporting Checklist: The authors have completed the CARE reporting checklist. Available at http://dx.doi.org/10.21037/ gs-20-780

Peer Review File: Available at http://dx.doi.org/10.21037/gs20-780
Conflicts of Interest: All authors have completed the ICMJE uniform disclosure form (available at http://dx.doi. org/10.21037/gs-20-780). The authors have no conflicts of interest to declare.

Ethical Statement: The authors are accountable for all aspects of the work in ensuring that questions related to the accuracy or integrity of any part of the work are appropriately investigated and resolved. Written informed consent was obtained from the patient for publication of this Case report and accompanying images. All procedures performed in studies involving human participants were in accordance with the ethical standards of China-Japan Friendship Hospital and with the Helsinki Declaration (as 
revised in 2013).

Open Access Statement: This is an Open Access article distributed in accordance with the Creative Commons Attribution-NonCommercial-NoDerivs 4.0 International License (CC BY-NC-ND 4.0), which permits the noncommercial replication and distribution of the article with the strict proviso that no changes or edits are made and the original work is properly cited (including links to both the formal publication through the relevant DOI and the license). See: https://creativecommons.org/licenses/by-nc-nd/4.0/.

\section{References}

1. Dalamau J, Rosenfeld M. Overview of paraneoplastic syndromes of the nervous system. Waltham, MA: UptoDate, 2018.

2. Rosenfeld MR, Dalmau J. Diagnosis and management of paraneoplastic neurologic disorders. Curr Treat Options Oncol 2013;14:528-38.

3. Walker GV, Smith GL, Perkins GH, et al. Populationbased analysis of occult primary breast cancer with axillary

Cite this article as: Sun X, Liu J, Yang M, Huang L. Axillary mass in a woman with progressive exertional limbs numbness and fatigue: a case report. Gland Surg 2021;10(3):1154-1157. doi: $10.21037 / g s-20-780$ lymph node metastasis. Cancer 2010;116:4000-6.

4. Foroudi F, Tiver KW. Occult breast carcinoma presenting as axillary metastases. Int J Radiat Oncol Biol Phys 2000;47:143-7.

5. Darnell RB, Posner JB. Paraneoplastic Syndromes Involving the Nervous System. New Engl J Med 2003;349:1543-4.

6. Hughes RA. Peripheral neuropathy. BMJ 2002;324:466-9.

7. Graus F, Keime-Guibert F, Reñe R, et al. Anti-Huassociated paraneoplastic encephalomyelitis: analysis of 200 patients. Brain 2001;124:1138-48.

8. Halsted WS. The Results of Radical Operations for the Cure of Carcinoma of the Breast. Ann Surg 1907;46:1-19.

9. Baron PL, Moore MP, Kinne DW, et al. Occult breast cancer presenting with axillary metastases: updated management. Arch Surg 1990;125:210-4.

10. Blanchard DK, Farley DR. Retrospective study of women presenting with axillary metastases from occult breast carcinoma. World J Surg 2004;28:535-9.

11. Matsuoka K, Ohsumi S, Takashima S, et al. Occult breast carcinoma presenting with axillary lymph node metastases: follow-up of eleven patients. Breast Cancer 2003;10:330-4. 